\title{
Vitrification of Ovarian Tissue from Non-Human Primates*
}

\author{
Adriel Behn Brito ${ }^{1,2}$, Cinthia Távora de Albuquerque Lopes ${ }^{1,2}$, Ana Paula Ribeiro Rodrigues ${ }^{3}$, \\ José Ricardo de Figueiredo ${ }^{3}$, Sheyla Farhayldes Souza Domingues ${ }^{1,2}$ \& Regiane Rodrigues dos Santos ${ }^{1,2}$
}

\begin{abstract}
Background: One of the strategies to preserve genetic material from nonhuman primates (NHP) consists in the implementation of germplasm banks, for future application in reproductive biotechniques, as well as for biomedical research. Based on the success rates achieved in human, there is a prominent possibility to succeed also with NHP. However, studies with NHP are still scarce, especially regarding the cryopreservation of ovarian tissue.

Review: Neotropical non-human primates, especially males, have been used in research related to reproductive biotechniques in Brazil. Regarding research on female reproduction and ovarian tissue preservation, most studies were performed using domestic animals as models. Current concepts and controversies in the restoration of gametes in adult females does not exclude the needs to preserve ovarian tissue. Importantly, ovarian tissue can be collected and preserved even after the death of the donors, being applied when finding dead females. Furthermore, collection of ovarian biopsies is also feasible and will not affect reproductive function. Among the cryopreservation methods, the vitrification has been indicated due to practical logistic, as well as because it will avoid the formation of large intracellular ice crystals, and it is claimed that ovarian stromal damage will be decreased under vitrification. Considering the number of threatened primate species and the needs to preserve their habitat, but also their gametes, development of preservation protocols are needed. Among the procedures, vitrification appears as a practical method to be applied in the near future. Although a low number of studies is reported, most of them were performed in the recent years. In this context, this article reviews recent information on the vitrification of ovarian tissue of non-human primates. Due to the limited number of studies in these species, observed data are compared with the literature in domestic and human mammals.

Conclusion: Despite the advances summarized here, it is possible to conclude that there is still a need to improve procedures for the preservation of genetic material, including in post-mortem situations. The technique of vitrification, therefore, consists of a feasible and promising option. However, it is needed to study properly the methods of vitrification, as well as the introduction of new systems and supplements to minimize or avoid the cell damage caused by the cryopreservation procedure (physical and chemical).
\end{abstract}

Keywords: non-human primates, ovarian tissue, cryopreservation.

*Article based upon a Dissertation submitted by the first author in partial fulfillment of requirements for the PhD's degree. 'Laboratório de Biologia e Medicina de Animais Silvestres da Amazônia (BIOMEDAM) \& ²Programa de Pós-graduação em Ciência Animal, Universidade Federal do Pará (UFPA), Castanhal, PA, Brazil. ${ }^{3}$ Laboratório de Manipulação de Oócitos e Folículos Ovarianos Pré-antrais, Universidade Estadual do Ceará (UECE), Fortaleza, CE, Brazil. CORRESPONDENCE: R.R. Santos [r.rodriguesdossantos@ @pq.cnpq.br - Tel.: +55 (91) 3213-0441]. Seção de Laboratórios, Centro Nacional de Primatas, Rodovia BR-316 km 7 s/n. Bairro Levilândia. CEP 67030-000 Ananindeua, PA, Brazil. 


\section{INTRODUÇÃO}

\section{CRONOLOGIA DA VITRIFICAÇÃO EM PRIMATAS NÃO- HUMANOS}

\section{PROTOCOLOS DE VITRIFICAÇÃO UTILIZADOS EM PRIMATAS NÃO-HUMANOS}

\section{PRINCIPAIS MÉTODOS DE ANÁLISE E FATORES QUE INFLUENCIAM O SUCESSO DA VITRIFICAÇÃO}

\section{CONCLUSÕES E PERSPECTIVAS}

\section{INTRODUÇÃO}

A implantação de bancos de germoplasma é essencial na manutenção de patrimônio genético e para sua utilização futura em biotécnicas reprodutivas. Ressalta-se a possibilidade de coleta e preservação de gametas após a morte de animais em risco de extinção [14,53], ou através de biópsias, sem prejuízo da função reprodutiva das doadoras [51].

Embora a renovação de gametas femininos em grandes mamíferos após o nascimento seja controversa [16], o ovário ainda é considerado uma fonte limitada de células reprodutivas que formam um pool de reserva representado pelos folículos pré-antrais (FOPA). Desta forma, a preservação de FOPA torna-se relevante e possível nessas espécies uma vez que o sistema reprodutivo feminino e a foliculogênese já foram caracterizados [1,12,29-31,58], bem como protocolos de maturação oocitária e de produção de embriões in vitro $[13,27]$ encontram-se disponíveis.

Apesar dos vastos estudos direcionados a animais domésticos, os avanços em primatas neotropicais ainda são recentes e geralmente focados na congelação convencional de sêmen $[25,26,41,42]$, com raros estudos sobre a criopreservação (congelação convencional ou vitrificação) de tecido ovariano [6].

A vitrificação em particular, vem sendo indicada pela praticidade de execução a campo, por evitar danos teciduais ocasionados por cristais de gelo e reduzir riscos de danos no estroma ovariano e foliculares [23]. Nos últimos cinco anos, publicações $[4,11,32,39,40,60,62]$ demonstraram os avanços da técnica (Figura 1). Desta forma, a presente revisão objetiva fornecer informações sobre o desenvolvimento cronológico da vitrificação em $\mathrm{PNH}$, métodos utilizados e principais resultados.
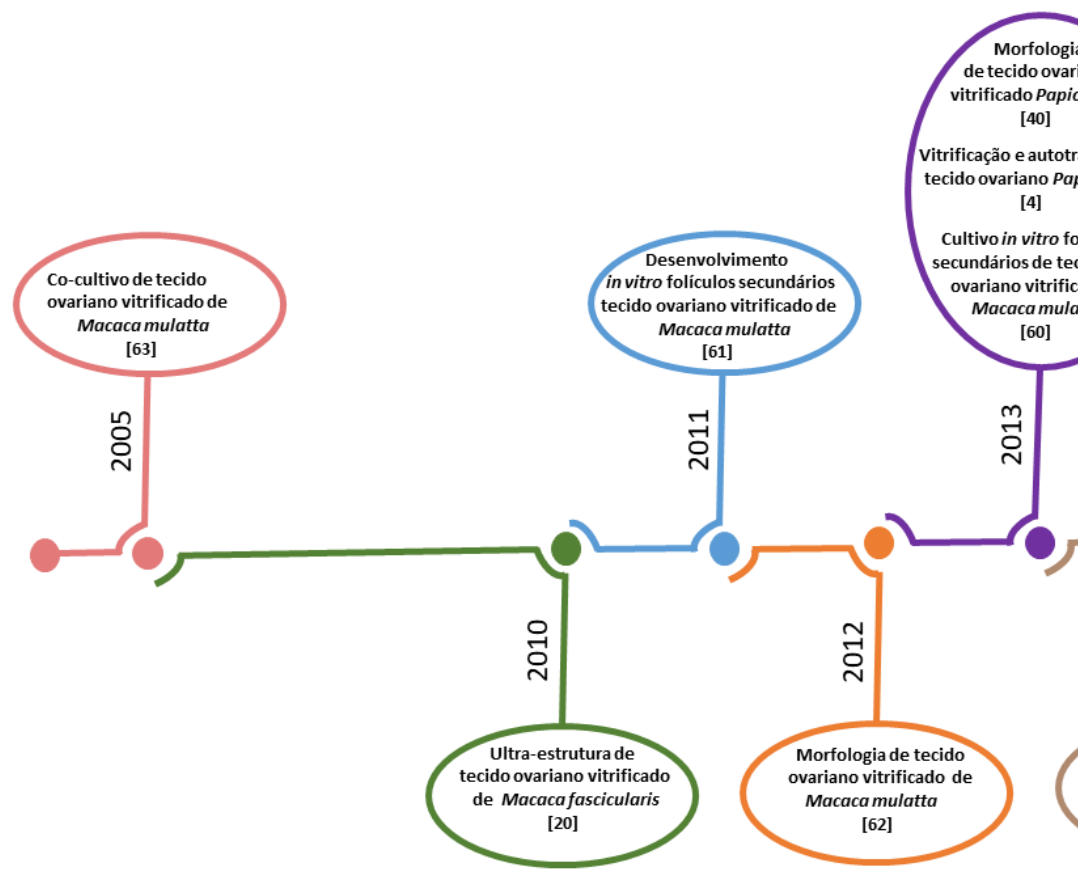

Mecido ovarian

vificado Papio anubis

[40]

Vitrificação e autotransplant

tecido ovariano Papio anubis

[4]

Cultivo in vitro folículos

secundários de tecido

ovariano vitrificado de

Vitrificação e transplante

de tecido ovariano de

Macaca fascicularis

Macaca mulatta [11]
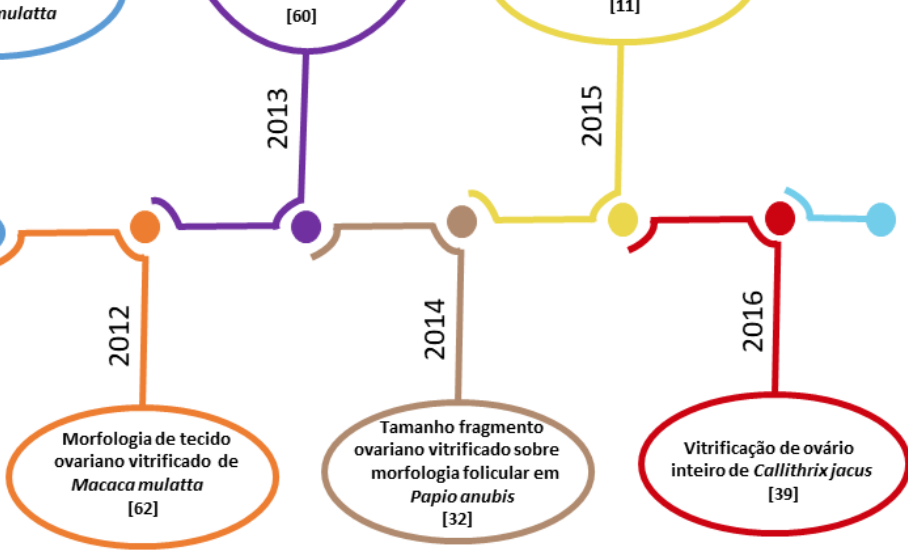

Figura 1. Ordem cronológica de publicações relacionadas à vitrificação de tecido ovariano de primatas não-humanos. 


\section{CRONOLOGIA DA VITRIFICAÇÃO EM PRIMATAS NÃO-HUMANOS}

Em 2005, foi publicado o primeiro relato de vitrificação de tecido ovariano de $\mathrm{PNH}$, no qual não foi observada diferença entre a congelação lenta e a vitrificação de tecido ovariano de Macaca mulata e Macaca fascicularis em relação às taxas de sobrevivência folicular [63]. Importante salientar que no citado trabalho a maioria dos folículos sobreviventes eram primordiais. Alguns anos depois, foi demonstrado que soluções de criopreservação complexas eram mais eficientes que soluções contendo apenas um crioprotetor para a vitrificação de folículos ovarianos de Macaca fascicularis, o que favorecia a manutenção da morfologia normal de mitocôndrias [20]. No ano seguinte, alguns autores indicaram ser possível, após vitrificação de tecido ovariano, obter o desenvolvimento in vitro de FOPA até a formação de antro [61]. Subsequentemente, os mesmos autores observaram que a suplementação do meio de vitrificação com polímeros sintéticos melhorava as taxas de sobrevivência folicular [62]. Infelizmente, esses resultados não foram substancialmente suficientes para otimizar o protocolo, e os autores observaram uma redução no diâmetro dos folículos vitrificados após cultivo in vitro por três semanas [60]. Posteriormente um promissor estudo piloto apresentou a preservação da morfologia folicular após o processo de vitrificação de tecido ovariano de babuínos [40].

Resultados mais animadores foram reportados ao auto-transplantarem tecido ovariano previamente vitrificado [4]. No estudo foi demonstrado que folículos primordiais de babuínos foram ativados e se desenvolveram nos transplantes, observando-se uma diminuição na taxa de folículos primordiais e aumento nas taxas de folículos secundários. Além disso, foi observada a presença de um corpo lúteo, indicando a ocorrência de ovulação. Em acréscimo, outro estudo sobre a vitrificação de tecido ovariano de babuínos, relatou a importância do tamanho do fragmento de ovário sobre a sobrevivência e funcionalidade folicular [32]. Após o cultivo in vitro dos fragmentos previamente vitrificados, a produção de estradiol foi maior em fragmentos de $1 \times 0,5 \times 1,5 \mathrm{~mm}$ do que em fragmentos de $1 \times 1 \times 2 \mathrm{~mm}$. Em seguida, foi demontrado que fragmentos ovarianos de Macaca fascicularis apresentavam melhor desenvolvimento folicular e qualidade estromal se vitrificados e auto-transplantados no local de um ovário imediatamente removido [11]. Recentemente, foi relatada a vitrificação de ovários inteiros de Callithrix jacchus [39], onde a proporção de folículos normais não diferiu da proporção observada no controle fresco. Contudo, houve uma redução na taxa de células somáticas viáveis após a vitrificação.

Apesar do número restrito de estudos em PNH, em comparação aos realizados em animais domésticos ou na própria espécie humana, é possível observar que alguns pesquisadores estão obtendo sucesso com o desenvolvimento de protocolos promissores. Desta forma, o envolvimento de pesquisadores especialistas em PNH com essa técnica de preservação de gametas, auxiliará na sua aplicação a campo permitindo a formação de bancos com valioso material genético. Além disso, as informações obtidas com diferentes protocolos de vitrificação e métodos de avaliação permitem o aprimoramento das técnidas de criopreservação a serem aplicadas em tecido ovariano de $\mathrm{PNH}$.

\section{PROTOCOLOS DE VITRIFICAÇÃO UTILIZADOS EM PRIMATAS NÃO-HUMANOS}

Apesar de ser um método simples, existem diferentes protocolos disponíveis de vitrificação. É importante ressaltar que não é objetivo da presente revisão detalhar técnicas, meios de vitrificação ou protocolos, o que pode ser aprofundado em revisões já publicadas $[2,33]$. A Tabela 1 apresenta as diferentes técnicas empregadas para a preservação de FOPA de PNH. Quando necessário outras técnicas desenvolvidas para grandes mamíferos domésticos, bem como para humanos, também são apresentadas. Com base na Tabela 1 é possível perceber a impossibilidade de comparar protocolos, devido à grande variação na composição dos meios de vitrificação (agentes crioprotetores - ACPs - e meios base), tempo e temperatura de exposição, e protocolos de aquecimento empregados pelos seis diferentes grupos de pesquisa aqui citados. A seguir, apenas alguns direcionamentos podem ser tomados.

O primeiro deles consiste no meio base, que possui uma constituição variável, sendo ou não suplementado com fontes proteicas, por exemplo, o substituto do soro ou albumina sérica humana. Além disso, a criopreservação requer a utilização de soluções contendo ACPs intracelulares, associados ou não com ACPs extracelulares. Dentre os ACPs intracelulares utilizados, o etilenoglicol (EG) está presente em todas as soluções de vitrificação, geralmente em combinação com dimetilsulfóxido (DMSO) ou glicerol. Na maioria das vezes, esses ACPs intracelulares estão associados 
a ACPs extracelulares como a sacarose $[4,11,39]$ e a polivinilpirrolidona (PVP) $[4,11,60,62]$. Outro açúcar que ainda não foi testado em primatas não-humanos é a trealose, que vem apresentando resultados animadores na vitrificação de tecido ovariano humano [59]. É importante considerar que outros tampões osmóticos [46] além de açúcares, deveriam ser considerados.

É comum vitrificar tecido ovariano com a utilização de apenas um crioprotetor intracelular como também com uma combinação de dois crioprotetores intracelulares. A eficiência do crioprotetor dependerá da ótima perfusão do tecido ovariano [37,45], o qual é complexo e fibroso em grandes mamíferos. Recentemente, foi demonstrado que a vitrificação de tecido ovariano ovino utilizando a combinação DMSO + EG resultou em uma redução na síntese de proteínas de canais transmembranares, as aquaporinas 3 e 9, o que não aconteceu quando a vitrificação foi realizada na presença de apenas EG com sacarose 0,25 M [49]. Independente da presença ou ausência da sacarose no meio de vitrificação, todos os estudos aqui revisados relatam a utilização da sacarose no meio de lavagem para remoção dos crioprotetores. Vitrificando tecido ovariano bovino, alguns autores mostraram que a adição de um crioprotetor extracelular como a sacarose no meio de remoção do crioprotetor pode aumentar as taxas de sobrevivência folicular [21], uma vez que o dano celular por choque osmótico é causado não apenas durante a exposição ao crioprotetor, mas também durante a sua remoção [56].

Existem estudos indicando que a temperatura de remoção do crioprotetor pode afetar a sobrevivência folicular pós-aquecimento. Por exemplo, foi observado que a remoção do crioprotetor realizada a 4,30 ou $37^{\circ} \mathrm{C}$ afetou as taxas de folículos ovarianos ovinos previamente criopreservados, sendo a temperatura de $30^{\circ} \mathrm{C}$ a mais indicada [43]. Contudo, pelos dados coletados em PNH, a temperatura de remoção do crioprotetor não tem sido um fator crucial para o sucesso do procedimento.

Diferentes técnicas de vitrificação comumente utilizadas são as denominadas de dropping (ou em gotas) [63], criotubos com agulhas no topo [20], lâmina de alumínio [61,62], agulhas [4,11], palhetas [40,60], vitrificação em superfície sólida - VSS [32], bem como cryotop [39]. A maioria dessas técnicas foi previamente testada em mamíferos de laboratório ou domésticos. Além dessas técnicas, um sistema que vem preservando com sucesso a qualidade folicular durante a vitrificação, consiste no OTC (ovarian tissue cryosystem em inglês). O primeiro relato foi publicado [9], e testes em tecido ovariano de $\mathrm{PNH}$ poderiam indicar se essa seria mais uma possibilidade a ser considerada nos procedimentos de vitrificação.

Tabela 1. Descrição de protocolos utilizados para a vitrificação de tecido ovariano de diferentes espécies de primatas não-humanos.

\begin{tabular}{|c|c|c|}
\hline \multirow[t]{2}{*}{ Espécie } & \multicolumn{2}{|l|}{ Descrição do protocolo } \\
\hline & Composição & Método \\
\hline $\begin{array}{c}\text { Macaca mulatta } \\
\text { [63] }\end{array}$ & 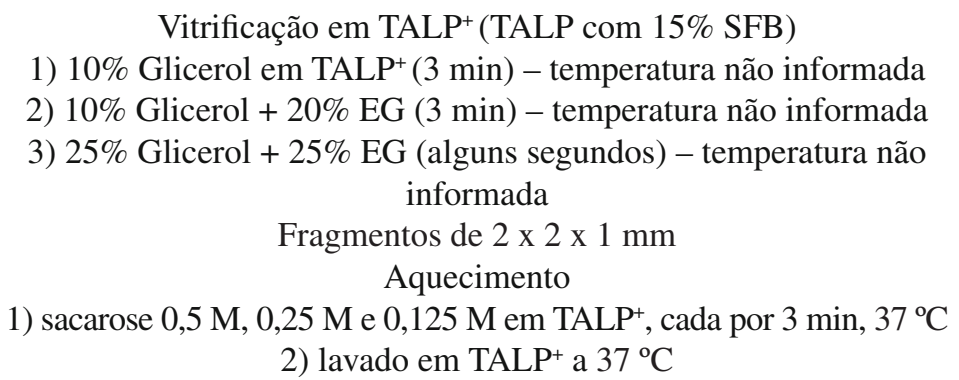 & $\begin{array}{l}\text { Dropping (gotas de } \\
\text { meio de vitrificação) }\end{array}$ \\
\hline
\end{tabular}


(...continuação)

\begin{tabular}{|c|c|c|}
\hline $\begin{array}{c}\text { Macaca } \\
\text { fascicularis [20] }\end{array}$ & 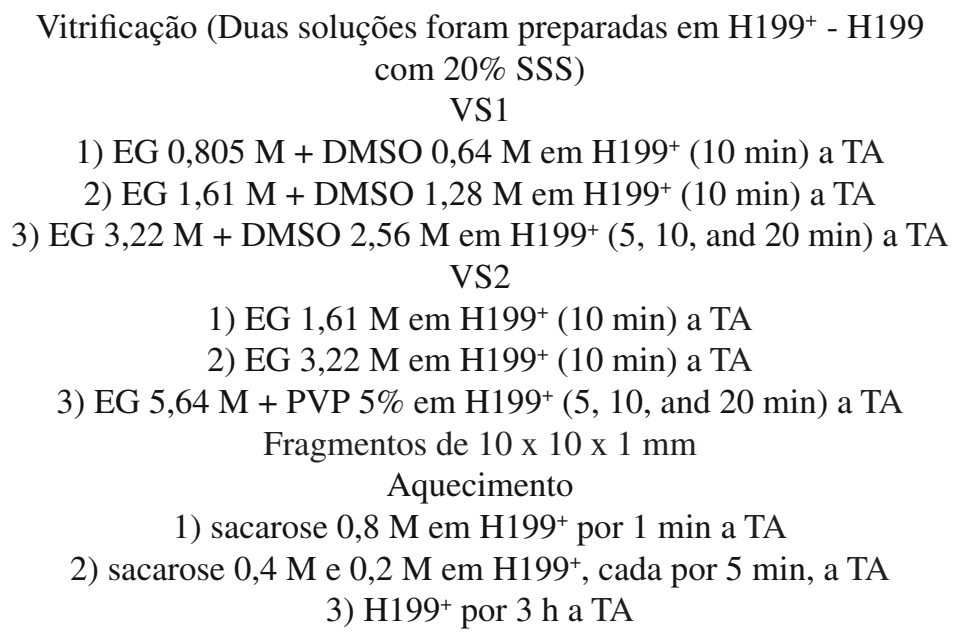 & $\begin{array}{l}\text { Criotubo com agulhas } \\
\text { no topo, a fim de } \\
\text { perfurar o tecido } \\
\text { ovariano. }\end{array}$ \\
\hline $\begin{array}{c}\text { Macaca mulatta } \\
{[61]}\end{array}$ & $\begin{array}{l}\text { Vitrificação em } \mathrm{HM}^{+} \text {(HM com } 15 \% \text { SPS e } 29 \mathrm{mg} / \mathrm{mL} \text { ácido } \\
\text { ascórbico }) \\
\text { 1) } 10 \% \text { Glicerol }(8 \mathrm{~min}) \text { a } 4{ }^{\circ} \mathrm{C} \\
\text { 2) } 10 \% \text { Glicerol }+20 \% \mathrm{EG}(8 \mathrm{~min}) \text { a } 4{ }^{\circ} \mathrm{C} \\
\text { 3) } 25 \% \text { Glicerol }+25 \% \mathrm{EG}(5 \mathrm{~min}) \text { a } 4{ }^{\circ} \mathrm{C} \\
\text { Fragmentos de } 3 \times 3 \times 1 \mathrm{~mm} \\
\text { Aquecimento } \\
\text { 1) sacarose } 0,5 \mathrm{M}, 0,25 \mathrm{M} \mathrm{e} 0,125 \mathrm{M} \text { em } \mathrm{HM}^{+} \text {, cada por } 5 \mathrm{~min} \text {, a } 37 \\
{ }^{\circ} \mathrm{C}\end{array}$ & $\begin{array}{c}\text { Lâmina de alumínio, } \\
\text { acondicionada em } \\
\text { Criotubos. }\end{array}$ \\
\hline $\begin{array}{c}\text { Macaca mulatta } \\
\text { [62] }\end{array}$ & 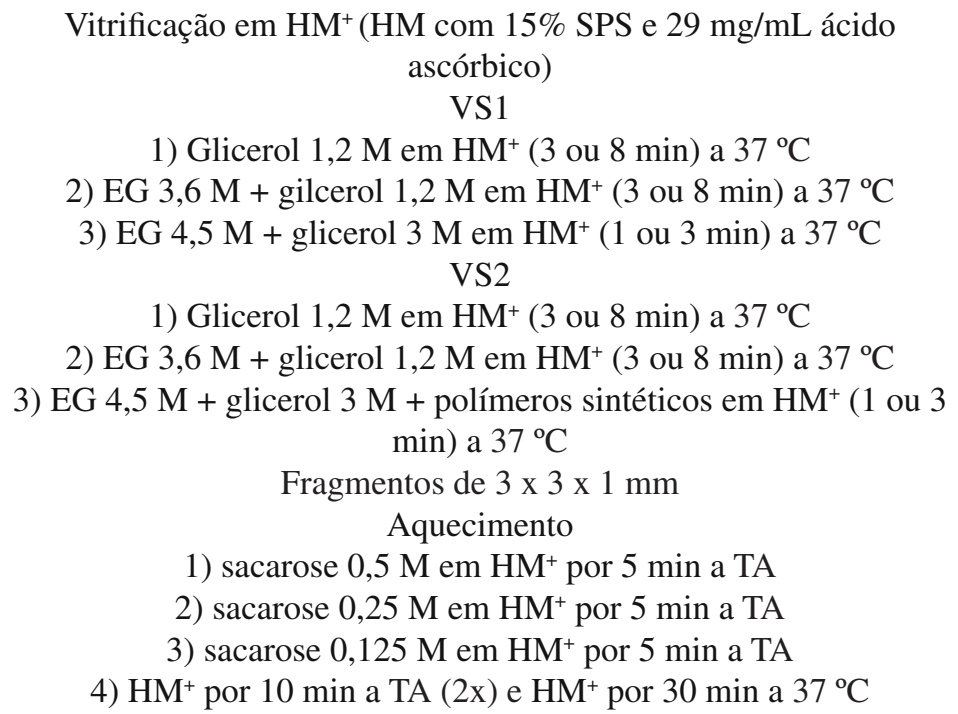 & $\begin{array}{c}\text { Lâmina de alumínio, } \\
\text { acondicionada em } \\
\text { Criotubos. }\end{array}$ \\
\hline Papio anubis [4] & 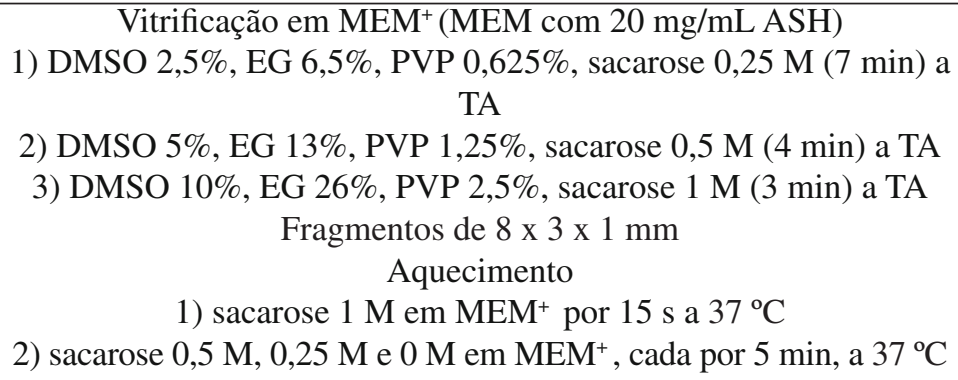 & Agulhas \\
\hline
\end{tabular}


(...continuação)

Vitrificação em $\mathrm{PBS}^{+}(\mathrm{PBS}$ com 10\% ASH)

1) DMSO 10\%, e EG $10 \%$ em $\mathrm{PBS}^{+}$por 2 min a TA

2) $\mathrm{DMSO} 20 \%$, e EG $20 \%$ em $\mathrm{PBS}^{+}$por $30 \mathrm{~s}$ a TA

Fragmentos de $1 \mathrm{~mm}^{3}$

Papio anubis [40]

$$
\text { Aquecimento }
$$

Palhetas $0,5 \mathrm{~mL}$

1) sacarose $0,5 \mathrm{M} \mathrm{em} \mathrm{PBS}^{+}$por $2 \mathrm{~min}$ a $37^{\circ} \mathrm{C}$

2) sacarose $0,25 \mathrm{M} \mathrm{em} \mathrm{PBS}^{+}$por $1 \mathrm{~min}$ a $37^{\circ} \mathrm{C}$

3) sacarose $0,125 \mathrm{M} \mathrm{em} \mathrm{PBS}^{+}$por $1 \mathrm{~min}$ a $37^{\circ} \mathrm{C}$

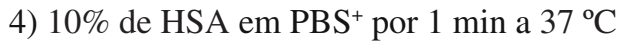

\begin{tabular}{|c|c|c|}
\hline $\begin{array}{c}\text { Macaca mulatta } \\
{[60]}\end{array}$ & $\begin{array}{l}\text { Vitrificação em } \mathrm{HM}^{+}(\mathrm{HM} \text { com } 15 \% \text { SPS e } 29 \mathrm{mg} / \mathrm{ml} \text { ácido } \\
\text { ascórbico }) \\
\text { VS1: EG } 27 \% \text { + glicerol } 27 \% \text { em } \mathrm{HM}^{+} \\
\text {VS2: EG } 25,5 \% \text { + DMSO } 25,5 \% \text { em } \mathrm{HM}^{+} \\
\text {1) } 25 \% \mathrm{VS} 1 \text { ou VS2 }(5 \mathrm{~min}) \text { a } 37^{\circ} \mathrm{C} \\
\text { 2) } 50 \% \text { VS1 ou VS2 }(5 \mathrm{~min}) \text { a } 37^{\circ} \mathrm{C} \\
\text { 3) } 100 \% \mathrm{VS} 1+\text { polímeros ou VS2 + polímeros }(1 \mathrm{~min}) \text { a } 37^{\circ} \mathrm{C} \\
\text { 4) } 1 \mathrm{~mL} \mathrm{VS1} \mathrm{+} \mathrm{polímeros} \mathrm{ou} 1 \mathrm{~mL} \text { VS } 2 \text { polímeros }(10 \mathrm{~min}) \text { ao } \\
\text { vapor de } \mathrm{NL}-\text { palhetas } \\
\text { Fragmentos de } 3 \times 3 \mathrm{~mm}^{2}\end{array}$ & $\begin{array}{c}\text { Palhetas } \\
(6 \times 65 \mathrm{~mm})\end{array}$ \\
\hline & 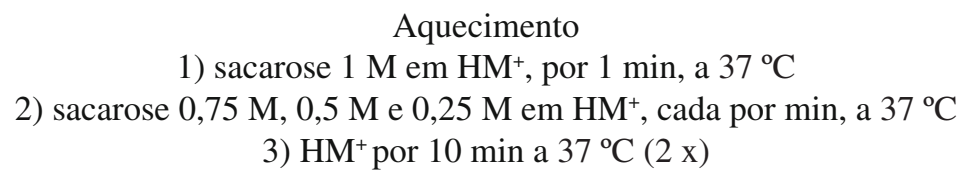 & \\
\hline
\end{tabular}

Vitrificação em $\mathrm{MEM}^{+}$(MEM com 25 mg/mL ASH)

1) $\mathrm{EG} 5 \%$ e DMSO $5 \%$ a $4{ }^{\circ} \mathrm{C}$

2) $\mathrm{EG} 10 \%$ e DMSO $10 \%$ a $4{ }^{\circ} \mathrm{C}$

3) EG $20 \%$ e DMSO $20 \%$ a $4{ }^{\circ} \mathrm{C}$

Tempo de exposição em cada etapa:

Papio anubis [32]

Fragmentos de $0,5 \times 1 \times 1 \mathrm{~mm}(2,5 ; 2,5 ; 5 \mathrm{~min})$

Fragmentos de 1 x 1 x $1 \mathrm{~mm}(5 ; 5 ; 10 \mathrm{~min})$

VSS

Fragmentos de 1 x 1 x $1,5 \mathrm{~mm}(7,5 ; 7,5 ; 15 \mathrm{~min})$

Fragmentos de 1 x 1 × 2 mm (10; 10;20 min)

Aquecimento

1) sacarose $1 \mathrm{M} \mathrm{em} \mathrm{MEM}^{+}$por $15 \mathrm{~s}$ a $37^{\circ} \mathrm{C}$

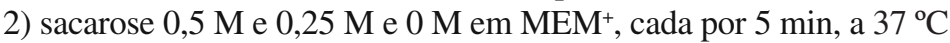

Vitrificação em $\mathrm{MEM}^{+}(\mathrm{MEM}+20 \mathrm{mg} / \mathrm{mL}$ ASH)

1) DMSO 2,5\%, EG 6,5\%, PVP 0,625\%, sacarose 0,25 M (4 min) a

TA

2) DMSO 5\%, EG 13\%, PVP 1,25\%, sacarose 0,5 M (4 min) a TA

Macaca 3) DMSO 10\%, EG 26\%, PVP 2,5\%, sacarose $1 \mathrm{M}$ (3 min) a TA

fascicularis [11]

Fragmentos de 5 x 1 x $1 \mathrm{~mm}$

Agulhas

Aquecimento

1) sacarose $1 \mathrm{M} \mathrm{em} \mathrm{MEM}^{+}$por $15 \mathrm{~s}$ a $37^{\circ} \mathrm{C}$

2) sacarose $0,5 \mathrm{M}, 0,25 \mathrm{M}$ e $0 \mathrm{M}$ em $\mathrm{MEM}^{+}$, cada concentração por

$5 \min$ a $37^{\circ} \mathrm{C}$

(continua...) 
(...continuação)

Vitrificação em TCM199+ (TCM199 Hepes com SSS 20\%)

1) $\mathrm{EG} 7,5 \%$ e DMSO $7,5 \%$ em TCM $199^{+}(15 \mathrm{~min})$ a TA

Callithrix jacus

[39]
2) EG $15 \%$, DMSO $15 \%$ e sacarose $0,5 \mathrm{M} \mathrm{em} \mathrm{TCM} 199^{+}(30 \mathrm{~min})$ a $4{ }^{\circ} \mathrm{C}$.

Ovário inteiro

Aquecimento

Cryotop

1) $\mathrm{TCM} 199^{+}$e sacarose $1 \mathrm{M}$ por 3 min a $37^{\circ} \mathrm{C}$.

2) $\mathrm{TCM}_{199^{+}}$e sacarose $0,5 \mathrm{M}$ por $5 \mathrm{~min}(\mathrm{TA})$

3) $\mathrm{TCM}_{199+}$ por $15 \mathrm{~min}(\mathrm{TA})-2 \mathrm{x}$

\section{PRINCIPAIS MÉTODOS DE ANÁLISE E FATORES QUE INFLUENCIAM O SUCESSO DA VITRIFICAÇÃO}

Para uma comparação subjetiva dos dados obtidos na literatura relacionada a $\mathrm{PNH}$, o presente tópico apresenta os métodos de análise utilizados nos dez trabalhos aqui apresentados, bem como os principais resultados obtidos (ver Tabela 2).

Em geral, a eficiência de todos os protocolos foi avaliada através de histologia clássica. Quando a morfologia folicular observada no tecido vitrificado não foi mantida similar àquela verificada no tecido fresco, as taxas de folículos normais atingiram cerca de 57\%. Análises realizadas imediatamente após a criopreservação geralmente não fornecem dados robustos relativos à eficiência do procedimento, uma vez que a qualidade folicular pode ser avaliada de maneira consistente após o cultivo in vitro ou transplante de tecido ovariano previamente criopreservado $[17,44]$. Dos dez estudos em PNH, três apresentam resultados imediatamente após o aquecimento das amostras de tecido e posterior remoção dos crioprotetores [20,39,40], cinco apresentam resultados após cultivo in vitro [32,60-63] e dois apresentam resultados após autotransplante $[4,11]$. Contudo, é necessário reconhecer que métodos eficientes de cultivo de FOPA ainda estão sendo desenvolvidos, especialmente para PNH. Além disso, o transplante do tecido ovariano também pode influenciar a sobreviência e o desenvolvimento folicular.
Relatou-se o desenvolvimento in vitro de folículos primários para o estágio de folículos secundários cultivados in vitro no tecido ovariano de Sapajus apella, por $24 \mathrm{~h} \mathrm{[5].} \mathrm{Contudo,} \mathrm{torna-se} \mathrm{necessário}$ ainda investigar se esse desenvolvimento folicular é sincrônico, i.e. se oócito e células da granulosa se desenvolvem de maneira conjunta permitindo o funcionamento normal de ambos os compartimentos foliculares, ou se os mesmos resultados seriam obtidos ao cultivar tecido ovariano previamente vitrificado. Além disso, em ovinos, foi observado que dependendo da fase folicular, a vitrificação deve ser realizada na forma de fragmentos ovarianos ou folículos isolados. Por exemplo, folículos secundários sobrevivem melhor se vitrificados isoladamente e não inclusos no tecido ovariano [35]. Não menos importante, a manutenção da estrutura tridimensional pode ser um fator facilitador para a sobrevivência e desenvolvimento folicular in vitro $[47,48]$.

Finalmente, o meio de criopreservação $[6,36]$ ou o meio de cultivo in vitro após aquecimento [19], tem permitido uma maior sobrevivência folicular [28,38]. A adição de antioxidantes e vitaminas para manter transplantes frescos ou criopreservados viáveis também tem sido relatada com sucesso [18,24,57], por reduzir a massiva ativação folicular e consequente perda do pool de reserva imediatamente após o transplante de tecido ovariano [54].

Tabela 2. Resumo de análises e resultados obtidos apos a vitrificação de tecido ovariano de diferentes espécies de primatas não-humanos.

\begin{tabular}{|c|c|c|}
\hline \multirow[t]{2}{*}{ Espécie } & \multicolumn{2}{|c|}{ Descrição } \\
\hline & Análises & Resultados \\
\hline $\begin{array}{l}\text { Macaca mulattae } \\
\text { M. fascicularis [63] }\end{array}$ & $\begin{array}{l}\text { Cultivo in vitro } 5 \text { dias } \\
\text { Viabilidade folicular (sonda fluorescente) }\end{array}$ & $\begin{array}{l}70 \% \text { folículos viáveis imediatamente após } \\
\text { vitrificação, e } 89 \% \text { folículos viáveis após } \\
\text { cultivo in vitro de tecido vitrificado. }\end{array}$ \\
\hline
\end{tabular}


(...continuação)

\begin{tabular}{|c|c|c|}
\hline $\begin{array}{c}\text { Macaca fascicularis } \\
{[20]}\end{array}$ & $\begin{array}{l}\text { Morfologia (histologia clássica - ultra- } \\
\text { estrutura) } \\
\text { Estimativa taxas de mitocôndrias intactas } \\
\text { Frequência de lisossomos }\end{array}$ & $\begin{array}{c}\text { Após aquecimento, 77-94\% folículos } \\
\text { normais. EG+DMSO superior a EG+PVP, } \\
\text { mas reduziu a percentagem de mitocôndrias } \\
\text { intactas e aumentou a frequência de } \\
\text { lisossomos. }\end{array}$ \\
\hline Macaca mulatta [61] & $\begin{array}{c}\text { Histologia clássica } \\
\text { Marcador de mitose (PPH3 e BrdU) } \\
\text { Quantificação de folículos secundários } \\
\text { Cultivo in vitro (5 semanas) de folículos } \\
\text { secundários } \\
\text { sobrevivência e crescimento folicular } \\
\text { Produção hormonal (E2, P4 e A4) }\end{array}$ & $\begin{array}{l}\text { Folículos primordiais, primários } \\
\text { e, especialmente, secundários bem } \\
\text { preservados. } 74 \% \text { folículos pré-antrais } \\
\text { normais. Após cultivo in vitro, proliferação } \\
\text { das células da granulosa do tecido } \\
\text { vitrificado foi similar ao obtido de tecido } \\
\text { fresco. Contudo, taxa de sobrevivência foi } \\
\text { inferior, bem como a formação de antro e } \\
\text { produção hormonal. }\end{array}$ \\
\hline Macaca mulatta [62] & $\begin{array}{c}\text { Histologia clássica } \\
\text { Marcador de mitose (PPH3) } \\
\text { Quantificação de folículos secundários } \\
\text { Cultivo in vitro (5 semanas) de folículos } \\
\text { secundários } \\
\text { Sobrevivência e crescimento folicular } \\
\text { Produção hormonal (E2, P4 e A4) }\end{array}$ & $\begin{array}{l}57 \% \text { e } 74 \% \text { de folículos normais foram } \\
\text { observados no tecido fresco, nos folículos } \\
\text { com } 2 \text { camadas e mais de } 2 \text { camadas, } \\
\text { respectivamente. Após cultivo in vitro, } \\
\text { proliferação das células da granulosa do } \\
\text { tecido vitrificado foi similar ao obtido } \\
\text { de tecido fresco. Conduto, taxa de } \\
\text { sobrevivência foi inferior, bem como a } \\
\text { formação de antro e produção hormonal. }\end{array}$ \\
\hline Papio anubis [4] & $\begin{array}{l}\text { Autotransplante } \\
\text { Histologia clássica } \\
\text { Densidade folicular } \\
\text { Imunohistoquímica } \\
\text { Detecção de fibrose }\end{array}$ & $\begin{array}{l}\text { Decréscimo na densidade da população } \\
\text { de folículos primordiais, e na proliferação } \\
\text { de células da granulosa dos folículos } \\
\text { secundários, mas sem efeitos no } \\
\text { funcionamento folicular. Vitrificação } \\
\text { aumentou a taxa de fibrose tecidual. Foi } \\
\text { observado corpo lúteo em todos os enxertos } \\
\text { após } 5 \text { meses. }\end{array}$ \\
\hline Papio anubis [40] & Histologia & $\begin{array}{l}84 \text { e } 67 \% \text { folículos normais foram } \\
\text { observados em tecido fresco e vitrificado, } \\
\text { respectivamente. }\end{array}$ \\
\hline Macaca mulatta [60] & $\begin{array}{c}\text { Histologia clássica } \\
\text { Marcador de mitose (BrdU) } \\
\text { Quantificação de folículos secundários } \\
\text { Cultivo in vitro (6 semanas) de folículos } \\
\text { secundários } \\
\text { Sobrevivência e crescimento folicular } \\
\text { Produção hormonal (E2, P4 e A4) }\end{array}$ & $\begin{array}{l}\text { Morfologia de folículos primordiais, } \\
\text { primários e secundários foi negativamente } \\
\text { afetada pela vitrificação combinando EG } \\
\text { e DMSO, quando comparada com EG + } \\
\text { glycerol. Após cultivo in vitro, (foi cultivado } \\
\text { apenas folículos secundários do grupo } \\
\text { EG + glycerol e do controle) proliferação } \\
\text { das células da granulosa e a sobrevivência } \\
\text { folicular foram similares para os obtidos de } \\
\text { tecidos frescos ou vitrificados. Conduto, a } \\
\text { taxa de a formação de antro foi inferior. A } \\
\text { produção de homônimo foi menor que no } \\
\text { controle, mas não teve diferença estatística. }\end{array}$ \\
\hline
\end{tabular}

\section{Macaca fascicularis} [20]

Estimativa taxas de mitocôndrias intactas

Frequência de lisossomos

\section{Marcador de mit}

Marcador de mitose (PPH

Quantificação de folículos secundários

secundários

sobrevivência e crescimento folicular

Produção hormonal (E2, P4 e A4)
Autotransplante

Detecção de fibrose (continua...) 


\begin{tabular}{|c|c|c|}
\hline Papio anubis [32] & $\begin{array}{c}\text { Histologia clássica } \\
\text { Marcador de apoptose (bax) } \\
\text { Cultivo in vitro (2 semanas) } \\
\text { Produção hormonal (E2, P4 e A4) }\end{array}$ & $\begin{array}{l}\text { Vitrificação reduziu as percentagens de } \\
\text { folículos morfologicamente normais } \\
\text { (Controle fresco } 92,6 \% \text { e tratamentos } \\
\text { de } 52,1 \% \text { a } 69,6 \% \text { de folículos } \\
\text { morfologicamente normais), reduziu a } \\
\text { produção hormonal in vitro, e aumentou as } \\
\text { taxas de apoptose. }\end{array}$ \\
\hline $\begin{array}{c}\text { Macaca fascicularis } \\
{[11]}\end{array}$ & $\begin{array}{l}\text { Autotransplante } \\
\text { Histologia clássica } \\
\text { Densidade folicular } \\
\text { Imunohistoquimica }\end{array}$ & $\begin{array}{l}\text { Decréscimo na densidade da população } \\
\text { de folículos pré-antrais, e na proliferação } \\
\text { das células da granulosa de folículos } \\
\text { secundários, mas sem efeitos no } \\
\text { funcionamento folicular. Vitrificação } \\
\text { aumentou a taxa de fibrose tecidual. }\end{array}$ \\
\hline Callithrix jacus [39] & $\begin{array}{c}\text { Histologia } \\
\text { Viabilidade (sonda fluorescente) } \\
\text { Integridade de DNA }\end{array}$ & $\begin{array}{c}\text { Densidade e morfologia folicular foram } \\
\text { similares quando comparando ovários } \\
\text { frescos e vitrificados. Houve diminuição } \\
\text { na sobrevivência dos folículos vitrificados } \\
\text { (86\% para 69\%). A integridade do DNA não } \\
\text { foi afetada pela vitrificação. }\end{array}$ \\
\hline
\end{tabular}

\section{CONCLUSÕES E PERSPECTIVAS}

Comparar resultados de diferentes grupos torna-se difícil quando variados métodos e pontos de observação são utilizados. Contudo, é possível observar que os avanços relacionados à vitrificação de tecido ovariano de PNH são promissores. Ainda faz-se necessário comparar diferentes técnicas de vitrificação em tecido ovariano de $\mathrm{PNH}$ antes de definir um protocolo a ser seguido na formação de bancos de germoplasma para essas espécies. Os crioprotetores EG e DMSO têm se mostrado os crioprotetores de escolha para a vitrificação de tecido ovariano, sendo utilizados de maneira isolada ou combinados. Apesar da adição de crioprotetores extracelulares como a sacarose ser rotineira, uma análise sobre a eficiência desse açúcar ainda é necessária. Além disso, outros métodos ou substâncias capazes de eliminar ou minimizar os danos causados pelo estresse oxidativo e osmótico precisam ser testados. Apesar das taxas de sucesso obtidas com a vitrificação de tecido ovariano serem animadoras, a sobrevivência e funcionalidade folicular após cultivo in vitro ou transplante do tecido ainda continuam sendo um desafio. Muitos dos danos causados serão observados apenas durante ou após a indução do desenvolvimento folicular. Para tanto, é necessário um maior aprofundamento sobre os mecanismos celulares e moleculares envolvidos na vitrificação, o desenvolvimento de protocolos específicos de cultivo in vitro, bem como o tratamento do tecido a ser transplantado para reduzir os danos causados por hipóxia pós-transplante.

Declaration of interest. The authors report no conflicts of interest. The authors alone are responsible for the content and writing of the paper.

Acknowledgements. Agradecemos à CAPES pela concessão de bolsa de doutorado para Adriel Behn Brito.

\section{REFERENCES}

1 Almeida D.V., Santos R.R., Scalercio S.R., Leão D.L., Haritova A., Oskam I.C. \& Domingues S.F. 2012. Morphological and morphometrical characterization, and estimation of population of preantral ovarian follicles from senile common squirrel monkey (Saimiri sciureus). Animal Reproduction Science. 134(3-4): 210-215.

2 Amorim C.A., Curaba M., Van Langendonckt A., Dolmans M.M. \& Donnez J. 2011. Vitrification as an alternative means of cryopreserving ovarian tissue. Reproductive Biomedicine Online. 23(2): 160-186.

3 Amorim C.A., Dolmans M.M., David A., Jaeger J., Vanacker J., Camboni A., Donnez J. \& Van Langendonckt A. 2012. Vitrification and xenografting of human ovarian tissue. Fertility and Sterility. 98(5): 1291-1298. 
4 Amorim C.A., Jacobs S., Devireddy R.V., Van Langendonckt A., Vanacker J., Jaeger J., Luyckx V., Donnez J. \& Dolmans M.M. 2013. Successful vitrification and autografting of baboon (Papio anubis) ovarian tissue. Human Reproduction. 28(8): 2146-2156.

5 Brito A.B., Santos R.R., Van den Hurk, R., Lima J.S., Miranda M.S., Ohashi OM \& Domingues S.F. 2013. Short-term culture of ovarian cortical strips from capuchin monkeys (Sapajus apella): a morphological, viability, and molecular study of preantral follicular development in vitro. Reproductive Sciences. 20(8): 990-997.

6 Brito D.C., Brito A.B., Scalercio S.R., Percario S. Miranda M.S., Rocha R.M., Diniz J.A., Oskam I.C., Van den Hurk R., Paris M.C., Domingues S.F. \& Santos R.R. 2014. Vitamin E-analog Trolox prevents endoplasmic reticulum stress in frozen-thawed ovarian tissue of capuchin monkey (Sapajus apella). Cell and Tissue Research. 355(2): 471-480

7 Brito D.C., Domingues S.F., Silva J.K., Wu X., Santos R.R. \& Pieczarka J.C. 2016. Detrimental effect of phenol red on the vitrification of cat (Felis catus) ovarian tissue. Biopreservation and Biobanking. 14(1): 17-22.

8 Carvalho A.A., Faustino L.R., Silva C.M., Castro S.V., Lobo C.H., Santos F.W., Santos R.R., Campello C.C., Bourdignon V., Figueiredo J.R. \& Rodrigues A.P. 2014. Catalase addition to vitrification solutions maintain goat ovarian preantral follicles stability. Research in Veterinary Science. 97(1): 140-147.

9 Carvalho A.A., Faustino L.R., Silva C.M., Castro S.V., Lopes C.A., Santos R.R., Bao S.N., Figueiredo J.R. \& Rodrigues A.P. 2013. Novel wide-capacity model for vitrification of caprine ovaries: ovarian tissue cryosystem (OTC). Animal Reproduction Science. 138(3-4): 220-227.

10 Celestino J.J., Santos R.R., Melo M.A., Rodrigues A.P. \& Figueiredo J.R. 2010. Vitrification of bovine ovarian tissue by the solid-surface vitrification method. Biopreservation and Biobanking. 8(4): 219-221.

11 Dolmans M.M., Binda M.M., Jacobs S., Dehoux J.P., Squifflet J.L., Ambroises J., Donnez J. \& Amorim C.A. 2015. Impact of the cryopreservation technique and vascular bed on ovarian tissue transplantation in cynomolgus monkeys. Journal of Assisted Reproduction and Genetics. 32(1): 1251-1262.

12 Domingues S.F., Almeida D.V., Scalercio S.R., Kastelic J.P. \& Santos R.R. 2011. Unilateral ovarian absence in two capuchin monkeys. Journal of Medical Primatology. 40(1): 37-40.

13 Domingues S.F., Caldas-Bussiere M.C., Petretski M.D., Ohashi O.M., Lima J.S., Santos R.R., Cordeiro M.S. \& Gomes de Castro P.H. 2010. Effects of follicular phase and oocyte-cumulus complexes quality on the protein profile and in vitro oocyte meiosis competence in Cebus apella. Fertility and Sterility. 93(5): 1662-1667. F.I.=4.373.

14 Domingues S.F.S., Lima J.S., Oliveira K.G. \& Santos R.R. 2011. Biotecnologias de reprodução como uma estratégia complementar à conservação in situ de primatas neotropicais ameaçados de extinção: perspectivas e desafios. Revista Brasileira de Reprodução Animal. 35(2): 124-129.

15 Faustino L.R., Santos R.R., Silva C.M., Pinto L.C., Celestino J.J., Campello C.C., Figueiredo J.R. \& Rodrigues A.P. 2010. Goat and sheep ovarian tissue cryopreservation: effects on the morphology and development of primordial follicles and density of stromal cell. Animal Reproduction Science. 122(1-2): 90-97.

16 Fereydouni B., Salinas-Riester G., Heistermann M., Dressel R., Lewerich L., Drummer C. \& Behr R. 2016. Long-term oocyte-like cell develoment in cultures derived from neonatal marmoset monkey ovary. Stem Cell. 2016: 2480-2498.

17 Figueiredo J.R., Rodrigues A.P., Silva J.R. \& Santos R.R. 2011. Cryopreservation and in vitro culture of caprine Preantral follicles. Reproduction, Fertility and Development. 23(1): 40-47.

18 Friedman O., Orvieto R., Fisch B., Felz C., Freud E., Ben-Haroush A. \& Abir R. 2012. Possible improvements in human ovarian grafting by various host and graft treatments. Human Reproduction. 27(2): 474-482.

19 Ganji R., Nabiuni M. \& Faraji R. 2015. Development of mouse preantral follicle after in vitro culture in a medium containing melatonin. Cell Journal. 16(4): 546-553.

20 Hashimoto S., Suzuki N., Yamanaka M., Hosoi Y., Ishizuka B. \& Morimoto Y. 2010. Effects of vitrification solutions and equilibration times on the morphology of cynomologus ovarian tissues. Reproductive Biomedicine Online. 21(4): 501-509.

21 Jimenez C.R., Penitente-Filho J.M., Torres C.A.A., Medeiros A.M. \& Silva L.S. 2016. Vitrification of bovine preantral follicles with dimethylsulfoxide and sucrose plus -tocopherol. Pesquisa Veterinária Brasileira. 36(3): 209-215.

22 Kawamura K., Cheng Y., Suzuki N., Deguchi M., Sato Y., Takae S., Ho C.H., Kawamura N., Tamura M., Hashimoto S., Sugishita Y., Morimoto Y., Hosoi Y., Yoshioka N., Ishizuka B. \& Hsueh A.J. 2013. Hippo signalling disruption and Akt stimulation of ovarian follicles for infertility treatment. Proceedings of National Academy of Science. 110(43): 17474-17479. 
23 Keros V., Xella S., Hultenby K., Pettersson K., Sheikhi M., Volpe A., Hreinsson J. \& Hovatta O. 2009. Vitrification versus controlled-rate freezing in cryopreservation of human ovarian tissue. Human Reproduction. 24(7): 1670-1683.

24 Kim E.J., Lee H.J., Lee J., Youm H.W., Lee J.R., Suh C.S. \& Kim S.H. 2015. The benefitial effects of polyethylene glycol-superoxide dismutase on ovarian tissue culture and transplantation. Journal of Assisted Reproduction and Genetics. 32(10): 1561-1569.

25 Leão D.L., Brito A.B., Miranda S.A., Oliveira K.G., Almeida D.V.C., Santos R.R. \& Domingues S.F. 2017. Extender supplementation with catalase maintains the integrity of sperm plasma membrane after freezing-thawing of semen from capuchin monkey. Zygote. 25(2): 231-234.

26 Leão D.L., Miranda S.A., Brito A.B., Lima J.S., Santos R.R. \& Domingues S.F. 2015. Efficacious long-term cooling and freezing of Sapajus apella semen in ACP-118®. Animal Reproduction Science159(1): 118-123.

27 Lima J.S., Leão D.L., Sampaio R.V., Brito A.B., Santos R.R., Miranda M.S., Ohashi O.M. \& Domingues S.F. 2013. Embryo production by parthenogenetic activation and fertilization of in vitro matured oocytes from Cebus apella. Zygote. 21(2): 162-166.

28 Lima-Verde I.B., Matos M.H.T., Bruno J.B., Martins F.S., Santos R.R., Bao S.N., Luque M.C.A., Vieira G.A.B., Silveira E.R., Rodrigues A.P.R., Figueiredo J.R., Oliveria M.A.L. \& Lima P.F. 2009. Effects of -tocopherol and ternatin antioxidants on morphology and activation of goat preantral follicles in vitro cultured. Arquivo Brasileiro de Medicina Veterinária e Zootecnia. 61(1): 57-65.

29 Lopes G.P., Brito A.B., Paim F.P., Santos R.R., Queiroz H.L. \& Domingues S.F. 2017. Comparative characterization of the external genitalia and reproductive tubular organs of three species of the genus Saimiri Voigt, 1831 (Primates: Cebidae). Anatomia, Histologia Embryologia. 46(2): 143-161.

30 Lopes G.P., Brito A.B., Santos R.R., Domingues S.F., Paim F.P. \& Queiroz H.L. 2017. Unilateral ovarian absence in a Black-headed squirrel monkey (Saimiri vanzolinii Ayres, 1985), a threatened neotropical primate species. Journal of Medical Primatology. 46(3): 87-89.

31 Lopes G.P., Santos R.R., Almeida D.V., Brito A.B., Queiroz H.L. \& Domingues S.F. 2017. Population estimate and morphometry of ovarian preantral follicles from three recently recognized squirrel monkey species: a comparative study. Zygote. 25(3): 279-287.

32 Lu X.L., Yu J., Zhang G., Wei Z.T., Li J.T. \& Zhang J.M. 2014. Effects of varying tissue sizes on the efficiency of baboon ovarian tissue vitrification. Cryobiology. 69(1): 79-83.

33 Lunardi F.O., Bass C.S., Bernuci M.P., Chaves R.N., Lima L.F., Silva R.F., Figueiredo J.R. \& Rodrigues A.P. 2015. Ewe ovarian tissue vitrification: a model for the study of fertility preservation in women. JBRA Assisted Reproduction. 19(4): 241-251.

34 Lunardi F.O., de Aguiar F.L., Apolloni L.B., Duarte A.B., de Sá N.A.R., Leal E.S.S., Sales A.D., Lobo C.H., Campello C.C., Smitz J., Apgar G.A., Figueiredo J.R. \& Rodrigues A.P. 2017. Sheep isolated secondary follicles are able to produce metaphase II oocytes after vitrification and long-term in vitro growth. Biopreservation and Biobanking. 15(4): 321-331.

35 Lunardi F.O., de Aguiar F.L., Duarte A.B., Araujo V.R., de Lima L.F., Ribeiro de Sa N.A., Vieira Correia H.H., Domingues S.F., Campello C.C., Smitz J. Figueiredo J.R. \& Rodrigues A.P. 2016. Ovine secondary follicles vitrified out the ovarian tissue grow and develop in vitro better than those vitrified into the ovarian fragments. Theriogenology. 85(7): 1203-1210.

36 Luz H.K., Santos R.R., Wanderley L.S., Faustino L.R., Silva C.M., Carvalho A.A., Campello C.C., Santos F.W., Figueiredo J.R. \& Rodrigues A.P. 2012. Catalase prevents lipid peroxidation and enhances survival of caprine preantral follicles cryopreserved in a 1,2-propanediol-freezing medium. Biopreservation and Biobanking. 10(4): 338-342.

37 Luz V.B., Santos R.R., Pinto L.C., Soares A.A., Celestino J.J., Mafezoli J., Campelo C.C., Figueiredo J.R. \& Rodrigues A.P. 2009. Dimethyl sulfoxide perfusion in caprine ovarian tissue and its relationship with follicular viability after cryopreservation. Fertility and Sterility. 91(4 Suppl): 1513-1515.

38 Melo M.A., Oskam I.C., Celestino J.J., Carvalho A.A., Castro S.V., Figueiredo J.R., Rodrigues A.P. \& Santos R.R. 2011. Adding ascorbic acid to vitrification and IVC medium influences preantral follicle mophology, but not viability. Reproduction in Domestic Animals. 46(4): 742-745.

39 Motohashi H.H. \& Ishibachi H. 2016. Cryopreservation of ovaries from neonatal marmoset monkeys. Experimental Animals. 65(3): 189-196. 
40 Nyachieo A., Spiessens C., Chai D.C., Kiulia N.M., Willemen D., Mwenda J.M., Bourgain C. \& D’Hooghe T.M. 2013. Ovarian tissue cryopreservation by vitrification in olive baboons (Papio Anubis): A pilot study. Gynecologic and Obstetric Investigation. 75(1): 157-162.

41 Oliveira K.G., Leão D.L., Almeida D.V., Santos R.R. \& Domingues S.F. 2015. Seminal characteristics and cryopreservation of sperm from the squirrel monkey, Saimiri collinsi. Theriogenology. 84(5): 743-749.

42 Oliveira K.G., Santos R.R., Leão D.L., Brito A.B., Lima J.S., Sampaio W.V. \& Domingues S.F. 2016. Cooling and freezing of sperm from captive, free-living and endangered squirrel monkey species. Cryobiology. 72(3): 283-289.

43 Oskam I.C., Asadi B.A. \& Santos R.R. 2010. Histologic and ultrastructural features of cryopreserved ovine ovarian tissue: deleterious effect of 1,2-propanediol applying different thawing protocols. Fertility and Sterility. 93(8): 27642766.

44 Oskam I.C., Lund T. \& Santos R.R. 2011. Irreversible damage in ovine ovarian tissue after cryopreservation in propanediol: analyses after in vitro culture and xenotransplantation. Reproduction in Domestic Animals. 46(5): 793-799.

45 Pinto L.C., Santos R.R., Faustino L.R., Silva C.G.M., Luz V.B., Maia Junior J.E., Soares A.A.X., Celestino J.J.H., Mafezoli J., Campello C.C., Figueiredo J.R. \& Rodrigues A.P. 2008. Quantification of dimethyl sulfoxide perfusion in sheep ovarian tissue: A predictive parameter for follicular survival to cryopreservation. Biopreservation and Biobanking. 6(1): 269-276.

46 Prasath E.B. 2008. Ovarian tissue cryopreservation: an update. Journal of Human Reproductive Science. 1(2): 50-55.

47 Rodrigues J.K., Navarro P.A.A.S., Zelinski M.B., Stouffer R.L. \& Xu J. 2015. Local role of androgens in the development of preantral follicles from nonhuman primates cultured individually a in three dimensional matrix. Reprodução \& Climatério. 29(3): 93-104.

48 Rodrigues J.K., Navarro P.A.A.S., Zelinski M.B., Stouffer R.L. \& Xu J. 2015. Direct actions of androgens on the survival, growth and secretion of steroids and anti-Müllerian hormone by individual macaque follicles during threedimensional culture. Human Reproduction. 30(3): 664-674.

49 Sales A.D., Duarte A.B., Santos R.R., Alves K.A., Lima L.F., Rodrigues G.Q., Brito I.R., Lobo C.H., Bruno J.B., Locatelli Y., Figueiredo J.R. \& Rodrigues A.P. 2016. Modulation of aquaporins 3 and 9 after exposure of ovine ovarian tissue to cryoprotectants followed by in vitro culture. Cell and Tissue Research. 365(2): 415-424.

50 Sanfilippo S., Canis M., Smitz J., Sion B., Darcha C., Janny L. \& Brugnon F. 2015. Vitrification of human ovarian tissue: a practical and relevant alternative to slow freezing. Reproductive Biology and Endocrinology. 13(1): 67.

51 Santana L.N., Brito A.B., Brito D.C., Lima J.S., Domingues S.F. \& Santos R.R. 2013. Adaptation of a trap door technique for the recovery of cortical biopsies from Cebus apella (capuchin monkeys). Zygote. 21(2): 158-161.

52 Santana L.N., Van den Hurk R., Oskam I.C., Brito A.B., Brito D.C., Domingues S.F. \& Santos R.R. 2012. Vitrification of ovarian tissue from primates and domestic ruminants: an overview. Biopreservation and Biobanking. 10(3): 288-294.

53 Santos R.R., Amorim C., Cecconi S., Fassbender M., Imhof M., Lornage J., Paris M., Schoenfeldt V. \& MartinezMadrid B. 2010. Cryopreservation of ovarian tissue: an emerging technology for female germline preservation of endangered species and breeds. Animal Reproduction Science. 122(3-4): 151-163.

54 Santos R.R., Knijn H.M., Vos P.L., Oei C.H., van Loon T., Colenbrander B., Gadella B.M., van den Hurk R. \& Roelen B.A. 2009. Complete follicular development and recovery of ovarian function of frozen-thawed, autotransplanted caprine ovarian cortex. Fertility and Sterility. 91(4 Suppl.): 1455-1458.

55 Santos R.R., Tharasanit T., Van Haeften T., Figueiredo J.R., Silva J.R. \& Van den Hurk R. 2007. Vitrification of goat preantral follicles enclosed in ovarian tissue by using conventional and solid-surface vitrification methods. Cell and Tissue Research. 327(1): 167-176.

56 Santos R.R., Van Haeften T., Roelen B.A., Knijn H.M., Colenbrander B., Gadella B.M. \& Van den Hurk. 2008. Osmotic tolerance and freezability of isolated caprine early-staged follicles. Cell and Tissue Research. 333(2): 323-331.

57 Scalercio S.R., Amorim C.A., Brito D.C., Percário S., Oskam I.C., Domingues S.F.S. \& Santos R.R. 2015. Trolox enhances follicular survival after ovarian tissue autograft in squirrel monkey (Saimiri collinsi). Reproduction, Fertility and Development. 28(11): 1854-1864.

58 Scalercio S.R., Brito A.B., Domingues S.F., Santos R.R. \& Amorim C.A. 2015. Immunolocalization of growth, inhibitory, and proliferative factors involved in initial ovarian folliculogenesis from adult common squirrel monkey (Saimiri collinsi). Reproductive Sciences. 22(1): 68-74. 
59 Tian T., Zhao G., Han D., Zhu K., Chen D., Zhang Z., Wei Z., Cao Y. \& Zhou P. 2015. Effects of vitrification cryopreservation on follicular morphology and stress relaxation behaviours of human ovarian tissues: sucrose versus trehalose as the non-permeable protective agent. Human Reproduction. 30(4): 877-883.

60 Ting A.Y., Yeoman R.R., Campos J.R., Lawson M.S., Mullen S.F., Fahy G.M. \& Zelinski M.B. 2013. Morphological and functional preservation of pre-antral follicles after vitrification of macaque ovarian tissue in a closed system. Human Reproduction. 28(5): 1267-1279.

61 Ting A.Y., Yeoman R.R., Lawson M.S., Zelinski M.B. 2011. In vitro development of secondary follicles from cryopreserved rhesus macaque ovarian tissue after slow-rate freeze or vitrification. Human Reproduction. 26(9): 2461-2472.

62 Ting A.Y., Yeoman R.R., Lawson M.S. \& Zelinski M.B. 2012. Synthetic polymers improve vitrification outcomes of macaque ovarian tissue as assessed by histological integrity and the in vitro development of secondary follicles. Cryobiology. 65(1): 1-11.

63 Yeoman R.R., Wolf D.P. \& Lee D.M. 2005. Coculture of monkey ovarian tissue increases survival after vitrification and slow-rate freezing. Fertility and Sterility. 83(Suppl 1): 1248-1254. 